\title{
Erratum: Super-micron-scale atomistic simulation for electronic transport with atomic vibration: Unified approach from quantum to classical transport [Phys. Rev. B 96, 035428 (2017)]
}

Keisuke Ishizeki, Kenji Sasaoka, Satoru Konabe, Satofumi Souma, and Takahiro Yamamoto (Received 15 December 2017; published 29 December 2017)

DOI: 10.1103/PhysRevB.96.239908 by

There are a few typographical errors for equations in the original paper. Equation (19) in the original paper should be replaced

$$
\vec{\Psi}_{+, \epsilon_{\mathrm{F}}, n}^{\mathrm{R}_{1(2)}}\left(t_{l}\right)=\frac{1}{N} \sum_{m=0}^{N-1} \exp \left(i \frac{2 \pi m}{N} l\right) \vec{\Phi}_{+, \epsilon_{\mathrm{F}}, n}^{\mathrm{R}_{1(2)}}\left(\omega_{m}\right),
$$

where $l$ is a discrete-time index, $N\left[=\left(t-t_{0}\right) / \Delta t\right]$ is the number of time steps for calculating the steady-state current $j_{\epsilon, n}^{\text {out }}$ defined by Eq. (6) in the original paper, and $\omega_{m}$ is an angular frequency labeled by a frequency index $m$.

Due to Eq. (19), Eqs. (20) and (21) in the original paper also should be replaced by

$$
\begin{aligned}
j_{\epsilon_{\mathrm{F}}, n}^{\text {out }} & =\sum_{m=0}^{N-1} J_{\epsilon_{\mathrm{F}}, n}\left(\omega_{m}\right), \\
J_{\epsilon_{\mathrm{F}}, n}\left(\omega_{m}\right) & \equiv \frac{2 a}{N \hbar} \operatorname{Im}\left[\left(\frac{1}{N} \vec{\Phi}_{+, \epsilon_{\mathrm{F}}, n}^{\mathrm{R}_{2}}\left(\omega_{m}\right)\right)^{\dagger} H^{\mathrm{R}_{2}, \mathrm{R}_{1}}\left(\frac{1}{N} \vec{\Phi}_{+, \epsilon_{\mathrm{F}}, n}^{\mathrm{R}_{1}}\left(\omega_{m}\right)\right)\right] .
\end{aligned}
$$

Numerical data shown in the original paper have been calculated by above equations. Thus, these typographical errors do not affect the results and conclusions of the original paper. 